\title{
Thermometric- and Acoustic-Based Beam Power Monitor for Ultra-Bright X-Rays
}

\author{
Gregory Bentsen \\ Office of Science, Science Undergraduate Laboratory Internship (SULI) \\ University of Rochester \\ SLAC National Accelerator Laboratory \\ Stanford, CA
}

August 14, 2009

Prepared in partial fulfillment of the requirements of the Office of Science, Department of Energy's Science Undergraduate Laboratory Internship under the direction of Joseph Frisch at the LCLS Diagnostics Group, SLAC National Accelerator Laboratory.

Participant:

Signature

Research Advisor:

Signature 


\section{TABLE OF CONTENTS}

$\begin{array}{ll}\text { Abstract } & \text { ii }\end{array}$

Introduction $\quad$ iii

Theory iv

Methods

Results

Discussion $\quad$ xiii

Conclusions $\quad$ XV

$\begin{array}{ll}\text { Acknowledgements } & \text { xvi }\end{array}$

References $\quad$ xix 


\begin{abstract}
Thermometric- and Acoustic-Based Beam Power Monitor for Ultra-Bright X-Rays. GREGORY BENTSEN (University of Rochester, Rochester, NY 14627)

JOSEPH FRISCH (LCLS Diagnostics Group, SLAC National Accelerator Laboratory, Stanford, CA 94025)

A design for an average beam power monitor for ultra-bright X-ray sources is proposed that makes simultaneous use of calorimetry and radiation acoustics. Radiation incident on a solid target will induce heating and ultrasonic vibrations, both of which may be measured to give a fairly precise value of the beam power. The monitor is intended for measuring ultrabright Free-Electron Laser (FEL) X-ray beams, for which traditional monitoring technologies such as photo-diodes or scintillators are unsuitable. The monitor consists of a Boron Carbide $\left(\mathrm{B}_{4} \mathrm{C}\right)$ target designed to absorb most of the incident beam's energy. Resistance temperature detectors (RTD) and piezoelectric actuators are mounted on the outward faces of the target to measure the temperature changes and ultrasonic vibrations induced by the incident beam. The design was tested using an optical pulsed beam (780 nm, 120 and $360 \mathrm{~Hz}$ ) from a Ti:sapphire oscillator at several energies between 0.8 and $2.6 \mathrm{~mJ}$. The RTDs measured an increase in temperature of about $10^{\circ} \mathrm{K}$ over a period of several minutes. The piezoelectric sensors recorded ringing acoustic oscillations at $580 \pm 40 \mathrm{kHz}$. Most importantly, the amplitude of the acoustic signals was observed to scale linearly with beam power up to $2 \mathrm{~mJ}$ of pulse energy. Above this pulse energy, the vibrational signals became nonlinear. Several causes for this nonlinearity are discussed, including amplifier saturation and piezoelectric saturation. Despite this nonlinearity, these measurements demonstrate the feasibility of such a beam power measurement device. The advantage of two distinct measurements (acoustic and thermometric) provides a useful method of calibration that is unavailable to current LCLS diagnostics tools.
\end{abstract}




\section{INTRODUCTION}

The Linac Coherent Light Source (LCLS) at the SLAC National Accelerator Laboratory is a new source of X-rays intended for probing molecular and atomic physics at very small length and time scales. Electrons accelerated and bunched by the existing linear accelerator are injected into undulators, which induce oscillations in the electron beam. The oscillating bunches radiate at a wavelength equal to the separation between bunches. The final X-rays have energies between $800 \mathrm{eV}$ and $9 \mathrm{keV}$. The pulse length of the beam is less than $10 \mathrm{fs}$, and is repeated at a rate of $10 \mathrm{~Hz}$. The beam diameter is about $100 \mu \mathrm{m}$. The energy per pulse is between 1 and $3 \mathrm{~mJ}$, resulting in a peak power of over $100 \mathrm{GW}$ and a peak brightness of over $10^{32}$ photons / $\mathrm{s} \mathrm{mm}^{2} \mathrm{mr}^{2}[10]$.

The exceptionally short pulse length and low emittance make the LCLS beam nearly ten billion times brighter than existing sources. As such, traditional devices and methods for measuring beam energy are not available for use in LCLS diagnostics. Photodiode circuits would, at best, be saturated by the high intensity of X-rays; at worst, they would be permanently damaged. Yttrium aluminum garnet (YAG) screens, which fluoresce when struck by incident X-ray or electron radiation, provide a possible alternative and are currently the primary diagnostic in use at LCLS [9] [14] [10]. Although intended to measure the beam's spatial profile and alignment, digital imaging processing may be applied to fluorescence images to obtain rough power measurements. Problems arise, however, when the beam energy increases above the screen's saturation point. In addition, calibration is tricky due to dependancies on the cameras used to capture the fluorescence, as well as degradation of the screen over time. Some studies have also shown inherent "blurriness" in YAG screen images that tends to enlarge the observed beam size, further reducing the viability of this technique as a precision beam power diagnostic [17].

The simplest alternative to these methods is calorimetry, a standard technique used in 
many detector applications. This technique is, in fact, already being used as one of the primary diagnostics tools for LCLS [8]. Calorimeters, however, suffer from complications of heat exchange and thermal noise, processes which are often difficult to either precisely quantify or eliminate. The proposed device aims to supplement the existing calorimetric technique with a less noisy alternative: radiation acoustics. Certain solids, when irradiated by a pulsed X-ray beam, will 'ring' ultrasonically, as if struck by a hammer [15]. These ultrasonic vibrations are relatively clean, have a narrow bandwidth $(Q>15)$, and have a ringing amplitude that varies linearly with beam energy [23]. This linear correlation may be used to determine beam energy based on a given measurement of ringing amplitude.

In order to use these phenomena in a measurement of beam energy, theoretical models were constructed. These were used both to provide proof of the device's feasibility as well as to give rough estimates of its responsiveness. Mechanical design and material choices were carried out based on the predictions of these models [16]. Design and testing of electronics were carried out alongside the mechanical design. A schematic drawing of the planned device

appears in Figure 1. A prototype device was machined and assembled with the intention of eventually installing a version in the LCLS beamline. The prototype was tested using an pulsed optical beam but was not installed.

\section{THEORY}

In order to compute a value for the beam power, theoretical models were constructed to characterize the system's thermal and acoustic properties. These models may then be used to solve for the beam energy given measurements of the thermal and acoustic excitation of the system. These models assume a $1 \mathrm{~cm}^{3}$ target composed of Boron Carbide. 


\section{Thermal Theory}

Validity of Lumped-system Model The following heat exchange model was constructed assuming a lumped system; this assumption posits that temperature gradients within objects may be ignored due to the small size and large thermal conductivity of the objects. This assumption is justified by the value of the Biot Number, $B i$, a dimensionless number that quantifies the relative rates of heating within and without an object:

$$
B i=\frac{h L}{k}
$$

where $h$ is heat transfer coefficient describing the rate of heating at a boundary, $L$ is the characteristic length of the object $(L \approx V o l . / A r e a)$, and $k$ is the thermal conductivity of the object. If $B i$ is much less than 1, the object internally equilibrates very quickly and temperature gradients may be ignored. The value of $B i$ for the proposed device was calculated to be 0.026 , indicating that temperature gradients within the $\mathrm{B}_{4} \mathrm{C}$ target are negligible.

Dynamic Heat Equation Simple heat exchange models were used to characterize the thermal behavior of the target after irradiation. To keep the models simple, the total heat flow to and from the $\mathrm{B}_{4} \mathrm{C}$ target was assumed to be approximately described by Newton's law of cooling. That is, the temperature of the target was assumed to be described by the

differential equation given by Equation (2), where solutions to the equation are given by Equation (3).

$$
\begin{gathered}
\frac{d T}{d t}=h\left(T-T_{a}\right)+k \\
T(t)=m e^{a t}+b
\end{gathered}
$$


$T$ is the temperature of the target and $h, k, m, a$, and $b$ are arbitrary coefficients. The ambient temperature, $T_{a}$, was assumed constant.

Five heat exchange processes were accounted for in the model: (a) heat flow through electrical wiring via the sensors; (b) convection currents in the ambient vacuum; (c) heat flow through the piezoelectric actuators; (d) exchange of thermal radiation between the target and the cylindrical beam line; and (e) exchange of thermal radiation between the target and tungsten collimator (labeled in Figure 1).

For processes (a) through (c), differential energy loss is governed by the same general equation as in Equation (2):

$$
\frac{d Q}{d t}_{T_{1} \rightarrow T_{2}}=h\left(T_{1}-T_{2}\right)
$$

where $\frac{d Q}{d t} T_{1} \rightarrow T_{2}$ is the thermal energy transferred between object 1 and $2, T_{1}$ and $T_{2}$ are the temperatures of the two objects, and $h$ is a constant governing the rate of heat exchange, determined by the dimensions and thermal properties of the system. These processes are thermally grounded to the ambient temperature $T_{a}$, so the variable $T_{2}$ may be replaced by the constant $T_{a}$ in Equation (4).

The radiative processes, (d) and (e), are governed by a different general equation:

$$
\frac{d Q}{d t}_{T_{1} \rightarrow T_{2}}=A_{1} \sigma\left(T_{1}^{4}-T_{2}^{4}\right) /\left[\frac{1}{\epsilon_{1}}+\frac{\left(1-\epsilon_{2}\right) A_{1}}{\epsilon_{2} A_{2}}\right]
$$

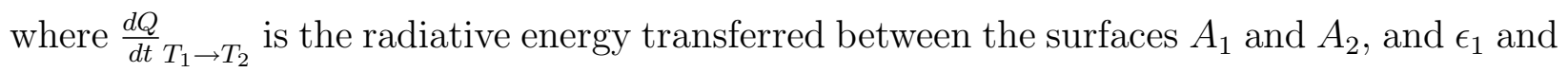
$\epsilon_{2}$ are the emissivities of the two surfaces, respectively. This equation may be approximated (via binomial expansion) by:

$$
\begin{gathered}
\frac{d Q}{d t}{ }_{T_{1} \rightarrow T_{2}, \text { approx. }}=h_{\text {rad. }}\left(T_{1}-T_{2}\right) \\
\text { where } \quad h_{\text {rad. }}=4 T_{2}^{3} A_{1} \sigma /\left[\frac{1}{\epsilon_{1}}+\frac{\left(1-\epsilon_{2}\right) A_{1}}{\epsilon_{2} A_{2}}\right] .
\end{gathered}
$$


In this way, one may quantify radiation exchange (i.e. processes (d) and (e)) in the simple form assumed in Equation (2).

Relative Significance of Heat Exchange Processes The relative significance of the processes (a) through (e) may be evaluated by comparing each process' "heat transfer coefficient," $h$. The larger this coefficient, the more heat energy is lost per unit time through a particular process. A plot of this comparison is shown in Figure 2. It is evident from this comparison that only heat loss through the piezoelectric actuators and radiation exchange with the beam line container are significant enough to warrant further consideration.

Total Heat Flow The total differential temperature change in the model is given by

$$
\frac{d T}{d t}_{\text {target }}=\frac{1}{m c_{v}}\left[\sum_{i=1}^{5} \frac{d Q_{i}}{d t}(T)+P_{\gamma}\right]
$$

where $\frac{d Q_{i}}{d t}(T)$ are the differential heat energies lost by each of the five processes, $P_{\gamma}$ is the power in the X-ray beam, and $m c_{v}$ is the target's specific heat. Solving for the temperature function $T(t)$, a limiting temperature of $301.2{ }^{\circ} \mathrm{K}$ and a time constant of $300 \mathrm{~s}$ were found (assuming a $10 \mathrm{~mW}$ average beam power). The theoretical temperature curve calculated with this model is shown in Figure 3.

Solving for Beam Power The heat losses $\frac{d Q_{i}}{d t}(T)$ are constant regardless of whether the beam is on or off. Therefore, these losses may be quantified when the beam is off and then filtered out when the beam is turned back on. The beam power is determined when these known heat losses have been corrected for. That is, given a temperature curve (like the one shown in Figure 3), one may solve for the characteristic time constant of the system using 
two points $\left\langle T_{1}, t_{1}\right\rangle$ and $\left\langle T_{2}, t_{2}\right\rangle$ on the "beam off" curve:

$$
\tau=\frac{t_{1}-t_{2}}{\log \left(T_{1}-T_{a}\right)-\log \left(T_{2}-T_{a}\right)}
$$

where $T_{a}$ is the ambient temperature (assumed to be $300^{\circ} \mathrm{K}$ in Figure 3 ). This time constant characterizes the heat losses in the system. Then the power of the beam may be solved for using this time constant and two points $\left\langle T_{3}, t_{3}\right\rangle$ and $\left\langle T_{4}, t_{4}\right\rangle$ on the "beam on" curve:

$$
P_{\gamma}=\frac{m c_{v}}{\tau}\left[T_{a}-\frac{T_{3}-T_{4} e^{\left(t_{3}-t_{4}\right) / \tau}}{1-e^{\left(t_{3}-t_{4}\right) / \tau}}\right]
$$

Complications Due to Target Clamp In the original heat exchange model, the large aluminum base supporting the target and collimators (see bottom of Figure 1) was assumed to remain at a fixed temperature, $T_{a}$, due to its large thermal mass. This assumption is not true, however, of the clamp (circled in red in Figure 1) that holds the target down on top of the aluminum mount. As the target's temperature increases, heat energy flows to the clamp, causing its temperature to rise as well. The dynamical flow of heat energy is impacted by this temperature change.

A more accurate model must consist of two coupled differential equations. This coupled system may be described by a system of matrices, analogous to the single-variable temperature equation given by Equation (2),

$$
\overrightarrow{\mathbf{T}^{\prime}}=[\mathbf{M}] \overrightarrow{\mathbf{T}}+\overrightarrow{\mathbf{b}}
$$

where $\overrightarrow{\mathbf{T}}$ is the temperature vector containing the two temperature values $T_{\text {target }}$ and $T_{\text {clamp }}$, $\overrightarrow{\mathbf{T}}^{\prime}$ is the differential temperature vector, $[\mathbf{M}]$ is the coupling matrix, and $\overrightarrow{\mathbf{b}}$ is the constant 
term vector. Solutions to this differential matrix equation are of the form:

$$
\begin{gathered}
\overrightarrow{\mathbf{T}}(t)=\overrightarrow{\mathbf{v}}[\mathbf{B}]\left[\begin{array}{cc}
e^{\lambda_{1} t} & 0 \\
0 & e^{\lambda_{2} t}
\end{array}\right][\mathbf{B}]^{-1}-[\mathbf{M}]^{-1} \overrightarrow{\mathbf{b}} \\
\text { where } \quad \overrightarrow{\mathbf{v}}=\overrightarrow{\mathbf{T}}_{\mathbf{i}}+[\mathbf{M}]^{-1} \overrightarrow{\mathbf{b}},
\end{gathered}
$$

$\overrightarrow{\mathbf{T}}_{\mathbf{i}}$ is the initial temperature vector, the columns of $[\mathbf{B}]$ are the eigenvectors of $[\mathbf{M}]$, and $\lambda_{1}$ and $\lambda_{2}$ are the eigenvalues of $[\mathbf{M}]$.

The results of this 2-variable model are shown in Figure 4. The "actual" temperature curve (i.e. temperature is affected by the clamp) is shown in blue, whereas the "nominal" curve (i.e. assuming the clamp has infinite thermal mass) is shown in brown. The clamp acts to retain heat in the system, resulting in higher limiting temperatures. Fortunately, since the second-order coupling effects are small, this variation may be compensated for by a simple multiplication factor. That is, the actual temperature curve is approximately proportional to the nominal temperature curve:

$$
T_{\text {act. }}(t)=\frac{1}{\kappa} T_{\text {nom. }}(t)
$$

where $\kappa=0.6622$ for all beam energies.

\section{Acoustic Theory}

Known theory may also be used to model acoustic phenomena. The generation of sound waves by X-ray radiation is considered in detail by Kalinichenko, et. al. [15]. For a photon beam, sound generation in a target is dominated by thermoelastic expansion due to lump energy deposition, as opposed to dynamic stresses from individual photons. In the range of X-ray energies produced by LCLS $(800 \mathrm{eV}-9 \mathrm{keV})$, energy is transferred to the target 
primarily via the photoelectric effect (as well as a smaller contributions from Rayleigh and Compton scattering). These effects generate secondary electrons, which continue to transfer energy to the target via ionization of target atoms. These mechanisms primarily increase the thermal energy of the target, causing thermal expansion that is responsible for wave generation.

For the vibrational measurement to have any significance, the fraction of beam energy absorbed by the target must be known. The most energetic X-rays in the FEL beam have energies less than $9 \mathrm{keV}$, giving a maximum attenuation length in $\mathrm{B}_{4} \mathrm{C}$ of about $2 \mathrm{~mm}$ [11]. Thus, roughly $95 \%$ of the incident photons have been absorbed in a target length of $6 \mathrm{~mm}$ (95\% length $\approx 3 \times$ atten. length). As a rough estimate, the attenuation length of the secondary electrons in the solid target may be given by the Bethe-Bloch formula,

$$
\left\langle\frac{d E_{e}}{d x}\right\rangle=-2 \pi\left(\frac{e^{2}}{4 \pi \epsilon_{0}}\right)^{2} \frac{z n}{E_{e}} \ln \left(\frac{\delta E_{e}}{I}\right)
$$

where $e$ is the electron charge, $z$ is the atomic number of the target material, $n$ is the atomic density, $I$ is the mean atomic potential, and $E_{e}$ is the initial electron energy [3]. Using this formula, the maximum stopping length for the most energetic electrons (i.e. $9 \mathrm{keV}$ ) may be calculated:

$$
d x=d E_{e} /\left[2 \pi\left(\frac{e^{2}}{4 \pi \epsilon_{0}}\right)^{2} \frac{z n}{E_{e}} \ln \left(\frac{\delta E_{e}}{I}\right)\right] \approx 6.6 \mu \mathrm{m} .
$$

Since the length of the target along the beam path is $10 \mathrm{~mm}$, it may be assumed that nearly all of the incident X-rays and secondary electrons are absorbed thermally by the target. That is,

$$
d E_{\text {target }} \approx E_{\text {pulse }} .
$$

Assuming that the target heats uniformly (see calculation of Biot number above), the amplitude of the thermoelastic wave may be approximately described as uniform expansion 
of the target in all dimensions due to heating. Using known values of specific heat $c_{v}$ and coefficient of thermal expansion $\alpha$ for Boron Carbide, the expected vibrational amplitude is:

$$
u=\frac{1}{2} \alpha L_{0} \frac{d E}{m c_{v}}
$$

where $u$ is the amplitude of vibration, $L_{0}$ is the initial length of the target, and $m c_{v}$ is the specific heat of the target. Given the assumption stated in Equation (14), the vibrational amplitude is then proportional to the pulse energy:

$$
\begin{gathered}
u=\zeta E_{\text {pulse }} \\
\text { where } \quad \zeta=\frac{\alpha L_{0}}{2 m c_{v}} .
\end{gathered}
$$

For the Boron Carbide target, $\zeta=11 \mathrm{pm} / \mathrm{mJ}$.

In addition, the resonant frequency of the target may also be calculated from the physical characteristics of the target:

$$
f=\frac{s}{2 L_{0}} \approx 545 \mathrm{kHz}
$$

where $s$ is the speed of sound in Boron Carbide and $L_{0}$ is the initial length of the target.

\section{METHODS}

Several proof-of-concept tests were carried out on a test target to determine the feasibility of the device and to verify theoretical calculations. A $0.8 \times 1 \times 1.25 \mathrm{~cm}^{3}$ Boron Carbide block was placed in the path of a pulsed $780 \mathrm{~nm}$ Q-switched Ti:sapphire beam. The target was oriented with the $1.25 \mathrm{~cm}$ face normal to the beam. The pulse length of the beam was roughly $10 \mathrm{~ns}$ at repetition rates of 120 and $360 \mathrm{~Hz}$. The bandwidth was $30 \mathrm{~nm}$, and the spatial profile was roughly Gaussian. Two monolithic ceramic piezoelectric actuators were 
glued to the target using Torr-Seal epoxy and clamped in place with an aluminum bar. Two 100 Ohm platinum RTDs were also mounted on the sides of the target.

The beam was attenuated and shaped by a wave plate and polarizer, and the spot size was controlled by a lens with a $0.5 \mathrm{~m}$ focus length. Before placing the target in the beam path, a power meter was used to measure the beam's average power. Two methods were used to vary the beam power: modification of the laser cavity length and attenuation via optical filters.

The signal from each piezoelectric actuator was fed to a 1:4 transformer, a $1.9 \mathrm{MHz}$ lowpass filter, a BJT preamplifier, and an oscilloscope. The oscilloscope was triggered on the beam pulse signal. Waveforms of the vibration signal were saved to disk for later analysis. The resistance (i.e. temperature) of the RTD was measured by a RTD temperature monitor using a 4-wire ratiometric measurement.

Several of the beam's parameters were varied over successive trials. The spot size was varied from 0.5 to $1 \mathrm{~cm} \varnothing$. The position of the beam on the target was varied from the bottom of the target to the top (roughly $4 \mathrm{~mm}$ off center in each case). The beam power was varied from $360 \mathrm{~mW}$ to $920 \mathrm{~mW}$ at $360 \mathrm{~Hz}$ repetition, and from $100 \mathrm{~mW}$ to $320 \mathrm{~mW}$ at $120 \mathrm{~Hz}$ repetition (these powers correspond to a range of roughly 0.8 to $2.6 \mathrm{~mJ}$ of pulse energy).

\section{RESULTS}

Plots of typical vibration signals from the irradiated block are shown in Figure 5. For low pulse energies $(\leq 2 \mathrm{~mJ})$, the amplitude of vibration was observed to vary linearly with beam power. This linearity disappears for pulse energies above $2 \mathrm{~mJ}$, where the vibrational

amplitude falls below the linear trend exhibited by the system at lower energies. A plot of vibration voltage versus beam power that exhibits this roll-off behavior is shown in Figure 
6. As seen in the figure, the magnitude of roll-off is dependent on the level of amplification, indicating that amplifier saturation may be to blame to for the observed nonlinearity. In an attempt to eliminate this effect, the amplifiers were removed from the system for three trials. This data is shown in Figure 7. Signal roll-off is still observed in these trials, indicating that factors other than amplifier saturation must contribute to nonlinearity in the vibration signal.

The resonant frequency of the block was observed to be $580 \pm 40 \mathrm{kHz}$, in good agreement with theory. When the diameter of the spot was halved, the vibrational amplitude was observed to increase by about 25\%. A detectable phase shift (on the order of $0.5 \mu \mathrm{s}$ ) was observed for trials in which the beam was aligned off-center. The RTDs detected a significant increase in temperature during each trial on the order of $10{ }^{\circ} \mathrm{C}$ after several minutes. This disagreed significantly with theory, which predicts a temperature rise of $43{ }^{\circ} \mathrm{C}$ over the same time period.

\section{DISCUSSION}

That the amplitude of vibration in fact varied linearly with beam power demonstrates the device's fundamental viability as a beam power monitor. It also places the device in

excellent agreement with similar experiments carried out by Kalinechenko and White [21] [23]. Unfortunately, the observed nonlinearity at high pulse energies is a significant problem. This effect could be due to several factors. The first could be a nonlinearity inherent in the piezoelectric actuators. The actuators may saturate above some maximum compression, resulting in reduced voltage signals for large compressions. Another, and more likely, source of disagreement stems from the properties of the beam used to test the device. While an X-ray beam penetrates through a good deal of the target material, inducing heating over a large depth, an optical laser is largely absorbed within a few micrometers, inducing large 
temperature gradients at the boundary of the target. The sound waves generated from this type of heating may differ significantly from those generated by the X-ray beam; this difference may be the cause of the unexpected nonlinearity.

In addition, the data disagrees with theory by a factor of 3 or more. A comparison of experimental data and theoretical predictions is shown in Figure 8. This disagreement could be due to several factors. The most sensible explanation is that the theoretical calculations do not account for resonances in the block. After irradiation, pressure waves must be expected to bounce around inside the target, constructively interfering to create compressions that are larger than those calculated from Equation (15). Another possible explanation considers again the differences between the FEL beam and the optical test beam. While theoretical calculations were carried out assuming a coherent, narrow $(\sim 100 \mu \mathrm{m})$ beam comprised of hard X-rays, the beam used to test the device was an optical beam with a much wider spot $(\sim 1 \mathrm{~cm})$. Since the details of pressure wave generation depend on the energy density and wavelength of incident radiation, these differing beam characteristics could have led to the creation of pressure waves with different amplitudes. Another suspected cause is a resonance in the cables that acted to amplify the piezoelectric signals they carried. These hypothesis must be investigated further in order to determine the true sources of discrepancy.

The dependence of vibration amplitude on spot size is also a significant impediment to the device's prospects as a power monitor. Ideally, the device should be able to measure average power regardless of the size of the spot, without any additional calibration. The literature, however, suggests that this effect is inherent to the phenomenon of wave generation by radiation beams. Calculations show that the peak vibrational amplitude depends critically on the beam radius as $u \propto R^{-3 / 2}$, where $u$ is the vibration amplitude and $R$ is the beam radius. Future work should focus on understanding and compensating for this effect.

The resonant frequency remained constant over all trials, in support of the simple wave propagation calculation shown in Equation (17). The stability of the resonant frequency 
is fortunate because it allows easy processing of the vibrational signal via either analog or digital filtering.

The phase shift in the vibration signal due to changes in beam position agrees with expectations based on the propagation of sound waves in Boron Carbide. The magnitude of phase difference, moreover, was large enough to justify using the device to make precision measurements of beam position in addition to its power measurement capabilities.

Although the behavior of the RTDs during irradiation qualitatively matched expectations, the amount of heating measured disagreed with expectations by nearly a factor of 5 . This difference may be explained by the dominant effect of convection currents in open air that acted to cool the device. In vacuum, such convection currents contribute to a negligible amount of cooling, resulting in the device reaching higher limiting temperatures. The device, however, must be tested in vacuum for the thermal technique to be truly verified.

\section{CONCLUSIONS}

As FEL X-ray sources reach higher brightness and peak power capabilities, new measurement techniques must be developed to withstand such high-power beams. The proposed device combines traditional calorimetry with the phenomenon of radiation acoustics in order to make a self-calibrated measurement of beam power. Such a technique may easily be adapted for use in future FELs with high beam brightness as well as in other pulsed sources such as hadron or electron beams.

Similar acoustic techniques may also be used to make precision measurements of other beam characteristics. These measurements include beam spot size, beam alignment, energy spectrum, spatial profile, and pulse duration. In addition, acoustic techniques may be used to study material properties such as mechanisms of radiation energy transfer, speed of sound, vibrational modes, and thermodynamic properties. Other applications include 
detection of solitary high-energy particles and modification of material properties during irradiation/acoustic wave generation [21].

Future studies must be carried out in order for the device to be considered ready for application. Theoretical studies should include understanding the discrepancies involved in the correlation between beam power and vibrational amplitude (especially with respect to the discussed nonlinearity) and more detailed models of wave generation and propagation in solids under irradiation. In addition, the application of radiation acoustics to beam position measurements must be quantified and studied further in order to use this technique with confidence.

\section{ACKNOWLEDGEMENTS}

This research was carried out at the SLAC National Accelerator Laboratory between May 18 and August 14, 2009 under the direction of Joseph Frisch. The author would like to thank the United States Department of Energy, the SLAC National Accelerator Laboratory, and the directors and administrators of the SULI internship program for making this research possible. He would also like to thank his mentors Joe Frisch and Tonee Smith as well as his partner Jennifer Loos. Thanks also go to Bill White, Steve Edstrom, and Ryan Coffee for their expertise and use of the NEH laser, Mark Petree and Phil Cutino for aid in mechanical design, Alan Fisher for guidance in electronics design, and Lesley Wolf for her indispensable help in acquiring research materials. 


\section{REFERENCES}

[1] A.F Adadurov and V.T Lazurik. Calculation of the yield of fast secondary electrons by the action of x radiation. Atomnaya Energiya, 50(3):211-212, 1981.

[2] R Akre, D Dowell, P Emma, J Frisch, S Gilevich, G Hays, Ph Hering, R Iverson, C Limborg-Deprey, H Loos, A Miahnahri, J Schmerge, J Turner, J Welch, W White, and J Wu. Commissioning the linac coherent light source injector. Phys. Rev. ST Accel. Beams, 11:30703, Mar 2008.

[3] H. Bethe. Zur theorie des durchgangs schneller korpuskularstrahlen durch materie. Annalen der Physik, 397(3):325-400, 1930.

[4] I.A Borshkovskii, V.D Volovik, I.A Grishaev, G.P Dubovik, I.I Zalyubovskii, and V.V Petrenko. Excitation of ultrasonic waves by passage of fast electrons through a metal. Zhurnal Eksperimentalnoi i Teoreticheskoi Fiziki, 13(10):546-549, Jul 1971.

[5] H.S. Carslaw and J.C. Jaeger. Conduction of Heat in Solids. Oxford University Press, 2nd edition, 1959.

[6] Alexander Wu Chao and Maury Tigner. Handbook of Accelerator Physics and Engineering. World Scientific Publishing Co., 1998.

[7] L. B da Silva, B. J MacGowan, S Mrowka, J. A Koch, R. A London, D. L Matthews, and J. H Underwood. Power measurements of a saturated yttrium x-ray laser. Optics Letters (ISSN 0146-9592), 18:1174, Jul 1993.

[8] S Friedrich, L Li, L. L Ott, Rajeswari M Kolgani, G. J Yong, Z. A Ali, O. B Drury, E Ables, and R. M Bionta. Design of a bolometer for total-energy measurement of the linear coherent light source pulsed x-ray laser. Nuclear Instruments and Methods in Physics Research Section A, 559:772, Apr 2006. 
[9] W. S Graves, E. D Johnson, and S Ulc. A high resolution electron beam profile monitor and its applications. The eighth beam instrumentation workshop. AIP Conference Proceedings, 451:206, Dec 1998.

[10] The LCLS Design Study Group. Linac coherent light source (lcls) design study report. SLAC Internal Report, 1998.

[11] B.L. Henke, E.M. Gullikson, and J.C. Davis. X-ray interactions: Photoabsorption, scattering, transmission, and reflection at $\mathrm{e}=50-30,000 \mathrm{ev}, \mathrm{z}=1-92$. Atomic Data and Nuclear Data Tables, 54(2):181-342, July 1993.

[12] Paul Horowitz and Winfield Hill. The Art of Electronics. Cambridge University Press, 2nd edition, 1989.

[13] Zhirong Huang and Kwang-Je Kim. Review of x-ray free-electron laser theory. Physical Review Special Topics - Accelerators and Beams, 10:34801, Mar 2007.

[14] E. D Johnson, W. S Graves, and K. E Robinson. Periscope pop-in beam monitor. The eighth beam instrumentation workshop. AIP Conference Proceedings, 451:479, Dec 1998.

[15] A.I. Kalinichenko, V.T. Lazurik, and I.I. Zalyubovsky. Introduction to Radiation Acoustics, volume 9 of The Physics and Technology of Particle and Photon Beams. Harwood Academic Publishers, 2001.

[16] Jennifer Loos. Design and implementation of a thermal and acoustic x-ray detector to measure the lcls beam energy. SLAC Internal Report, Aug 2009.

[17] A. H Lumpkin, B. X Yang, W. J Berg, M White, J. W Lewellen, and S. V Milton. Optical techniques for electron-beam characterizations on the aps sase fel project. Nuclear Instruments and Methods in Physics Research Section A, 429:336, Jun 1999. 
[18] John F. O'Hanlon. A User's Guide to Vacuum Technology. John Wiley Sons, Inc., 3rd edition, 2003.

[19] James Rosenzweig and Luca Serafini, editors. The Physics of High Brightness Beams. Proceedings of the 2nd ICFA Advanced Accelerator Workshop. World Scientific Publishing Co., 2000.

[20] Yoshihiko Shoji. Measurement of the time structure of a coherent synchrotron radiation burst in newsubaru. Infrared Physics \& Technology, 51:367, May 2008.

[21] V.D Volovik, A.I Kalinichenko, V.I Kobizskoi, and V.T Lazurik-El'tsufin. Thermoelastic effect of a fast particle in a solid. Zhurnal Eksperimentalnoi i Teoreticheskoi Fiziki, 19(2):135-138, 1974.

[22] X.J Wang, I Ben-Zvi, R Malone, and V Yakimenko. High-resolution beam profile monitor r\&d at the bnl atf. Brookhaven Internal Report, Sep 2000.

[23] R. M White. Elastic wave generation by electron bombardment or electromagnetic wave absorption. Journal of Applied Physics, 34:2123, Jul 1963. 


\section{FIGURES}

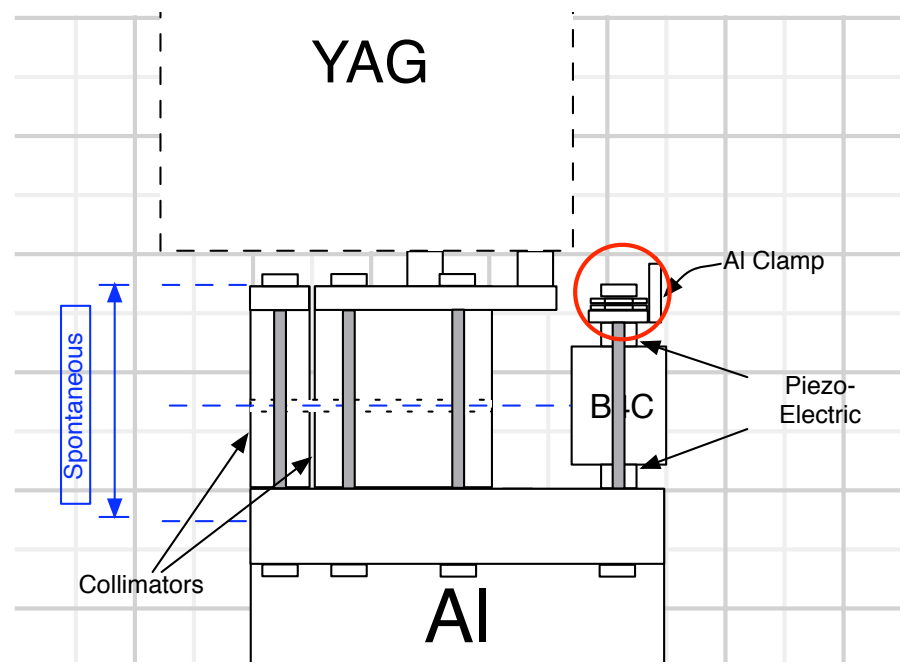

(a) View from the side of the device. The coherent spot passes through a slit in the Boron Carbide and Tungsten collimators to hit the $\mathrm{B}_{4} \mathrm{C}$ target.

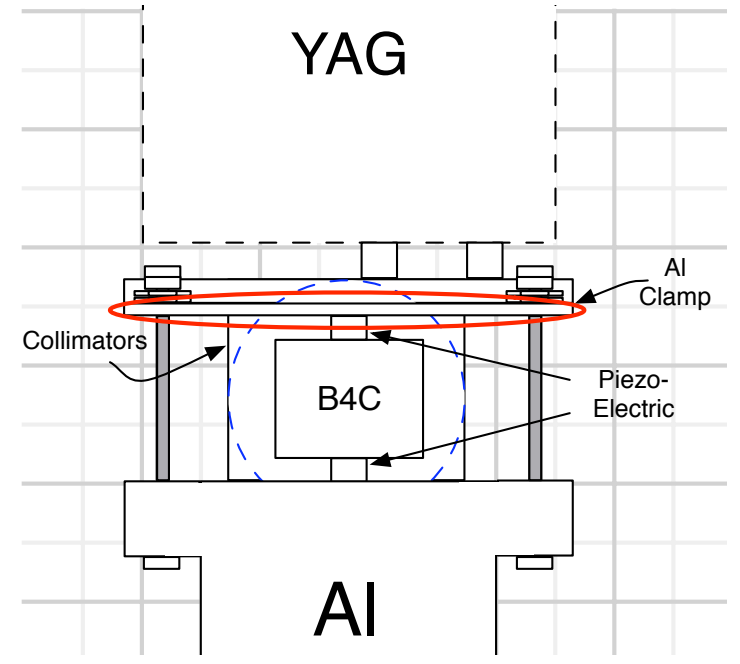

(b) View from the back of the device. The spontaneous radiation may be seen as the dotted blue line filling the front collimator.

Figure 1: (Color) Schematic diagrams of the proposed LCLS beam power monitor. Red circles mark the target clamp that gives rise to second-order thermal behavior in the system. 


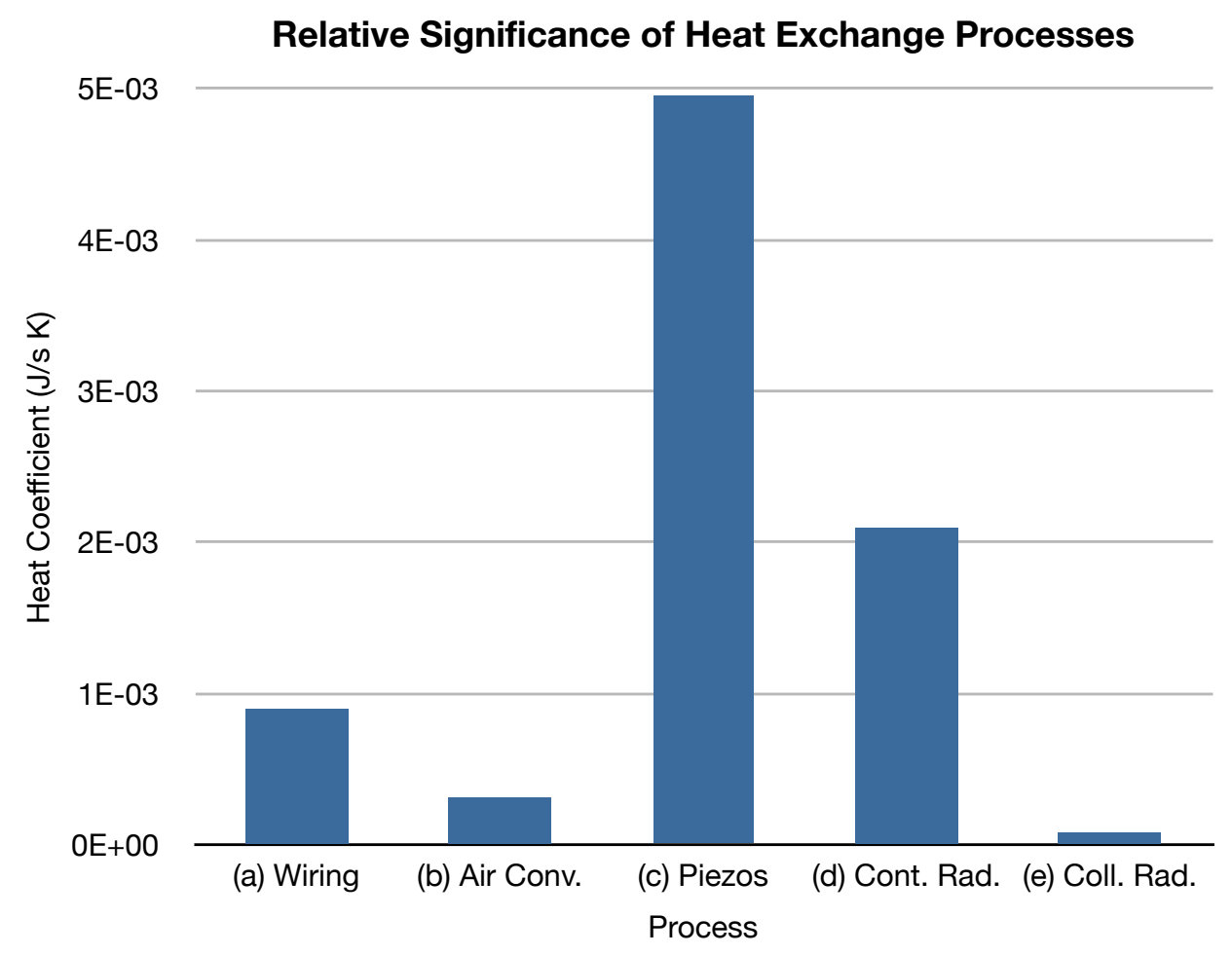

Figure 2: Relative significance of heat exchange processes in the power monitor system. Only the heat loss through the piezoelectric actuators and ambient radiation are significant enough to be regarded in future calculations. 
$\mathrm{T}(\mathrm{K})$

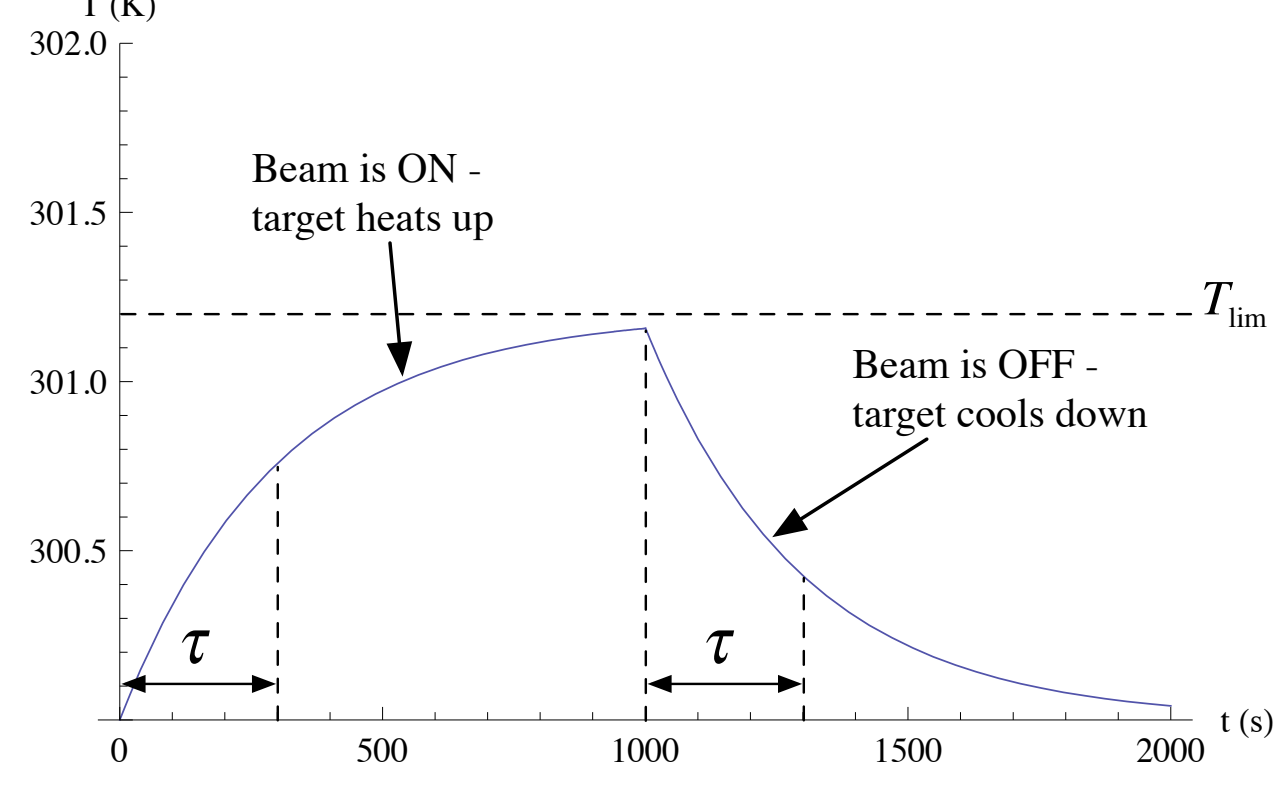

Figure 3: Theoretical temperature curve for a Boron Carbide target irradiated by a pulsed $\mathrm{X}$-ray beam. The beam is turned on at time $\mathrm{t}=0 \mathrm{~s}$, and is turned off at time $\mathrm{t}=1000 \mathrm{~s}$. The target is assumed to reside in an ambient temperature of $300{ }^{\circ} \mathrm{K}$. 


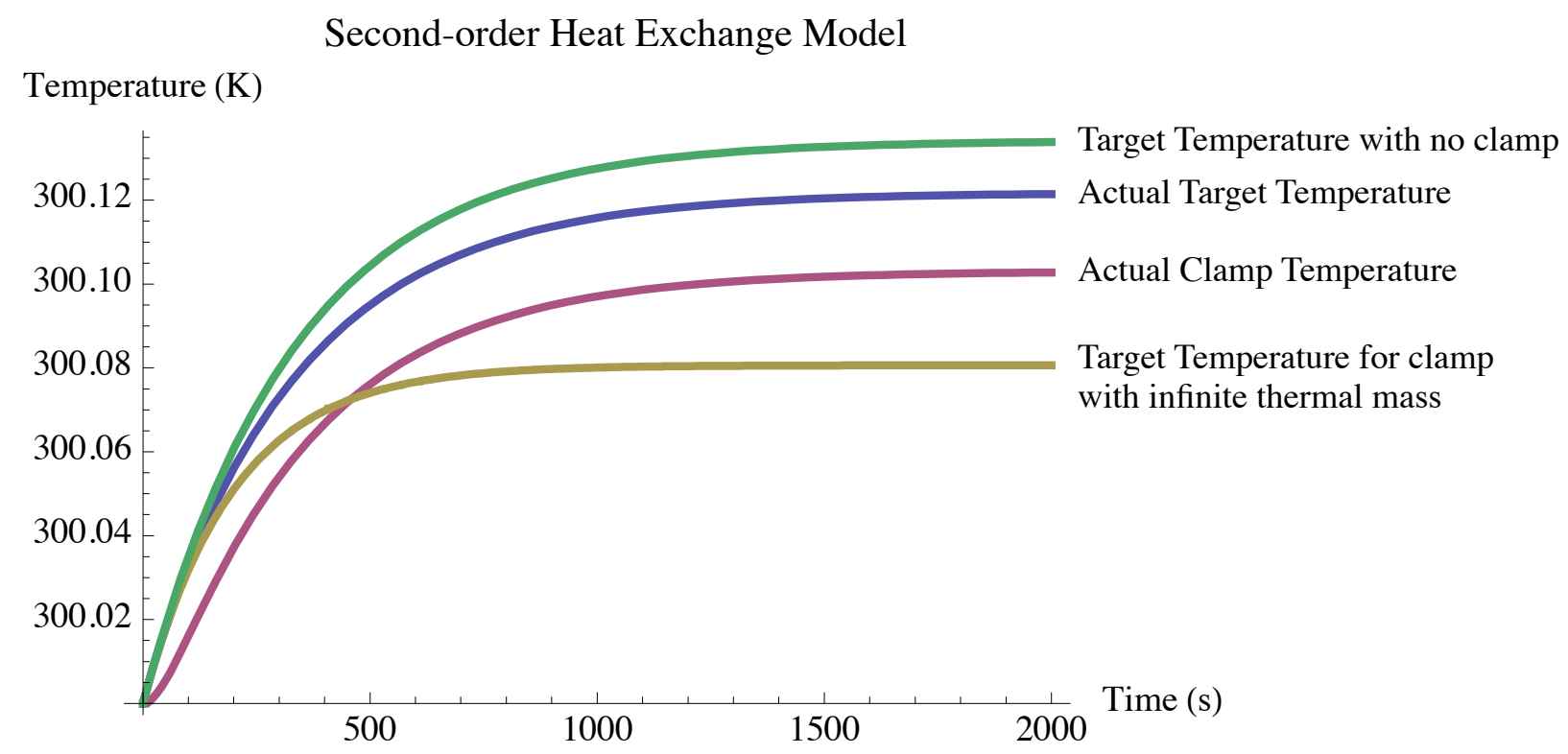

Figure 4: (Color) Temperature curves for the target and its associated clamp. The Blue and Red lines are the temperatures of the target and clamp, respectively. (Note the second-order behavior of the clamp between $t=0 \mathrm{~s}$ and $\mathrm{t}=100 \mathrm{~s}$ due to coupling effects). The Green line is the target's temperature curve if the target clamp were removed, and the Brown line is the target's temperature curve assuming a clamp with infinite thermal mass (i.e. the clamp remains at constant temperature regardless of the target's temperature). 


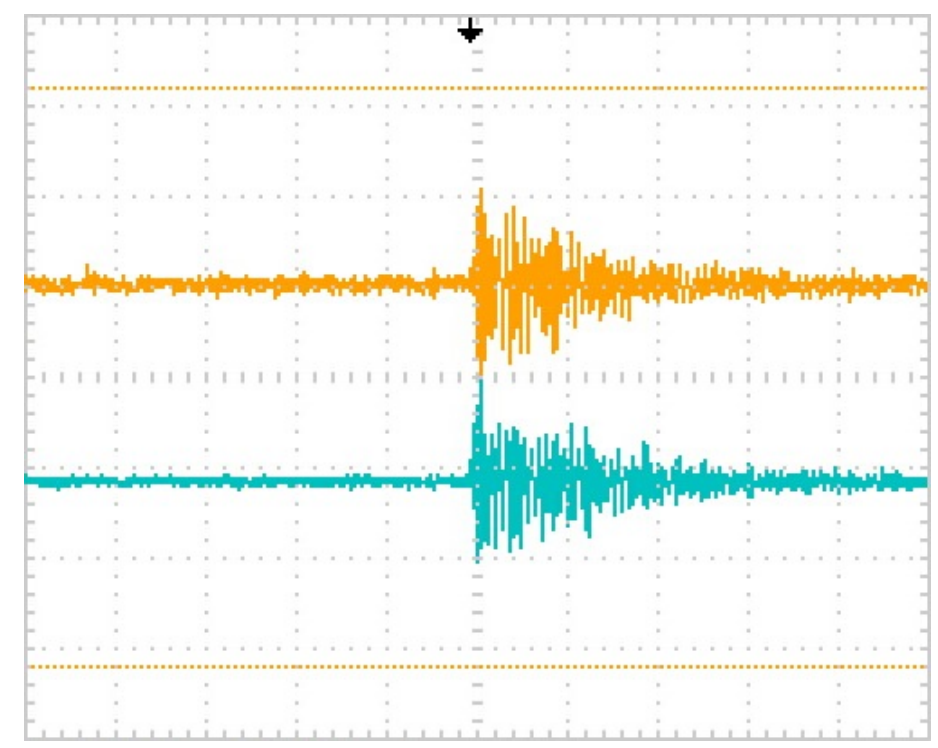

(a) Vibrational signals in irradiated test target. Units: 25 $\mu$ s/div. $\times 200 \mathrm{mV} /$ div.

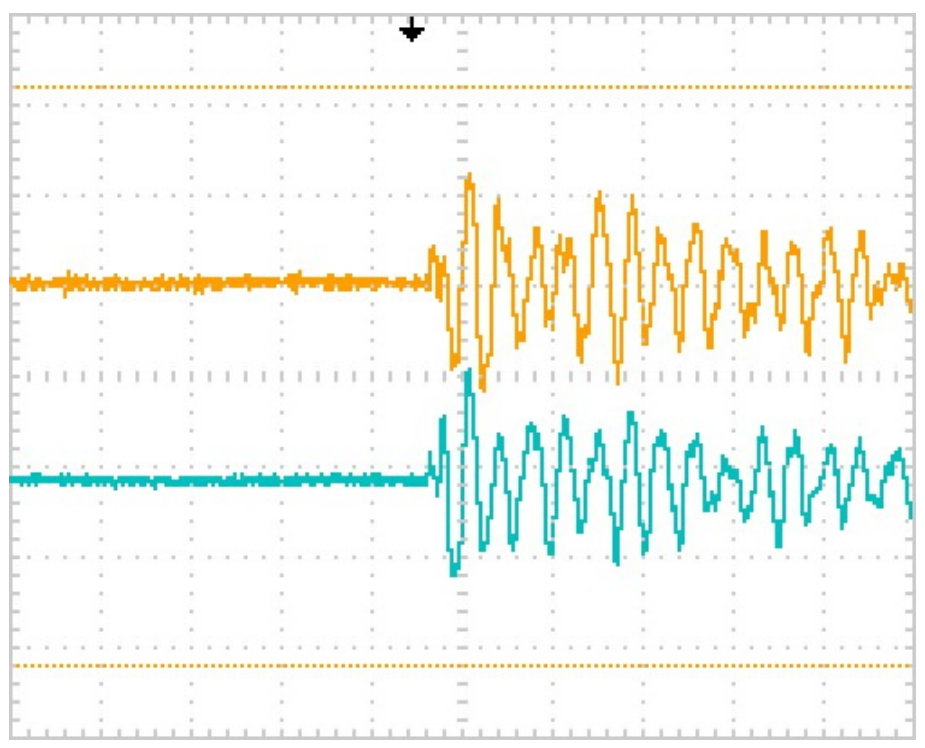

(b) Time detail of Figure (a). Units: $5 \mu \mathrm{s} /$ div. $\times 200 \mathrm{mV} /$ div.

Figure 5: (Color) Typical signals produced by radiation acoustic waves in the test target. Orange and Blue waveforms were generated by the two piezoelectric actuators mounted on either side of the target. Figure (b) shows time detail of Figure (a). Waveforms are triggered on the beam pulse. Note the exponential decay of vibration in Figure (a). 


\section{Vibrational Amplitude vs. Beam Power at various Amplifications}

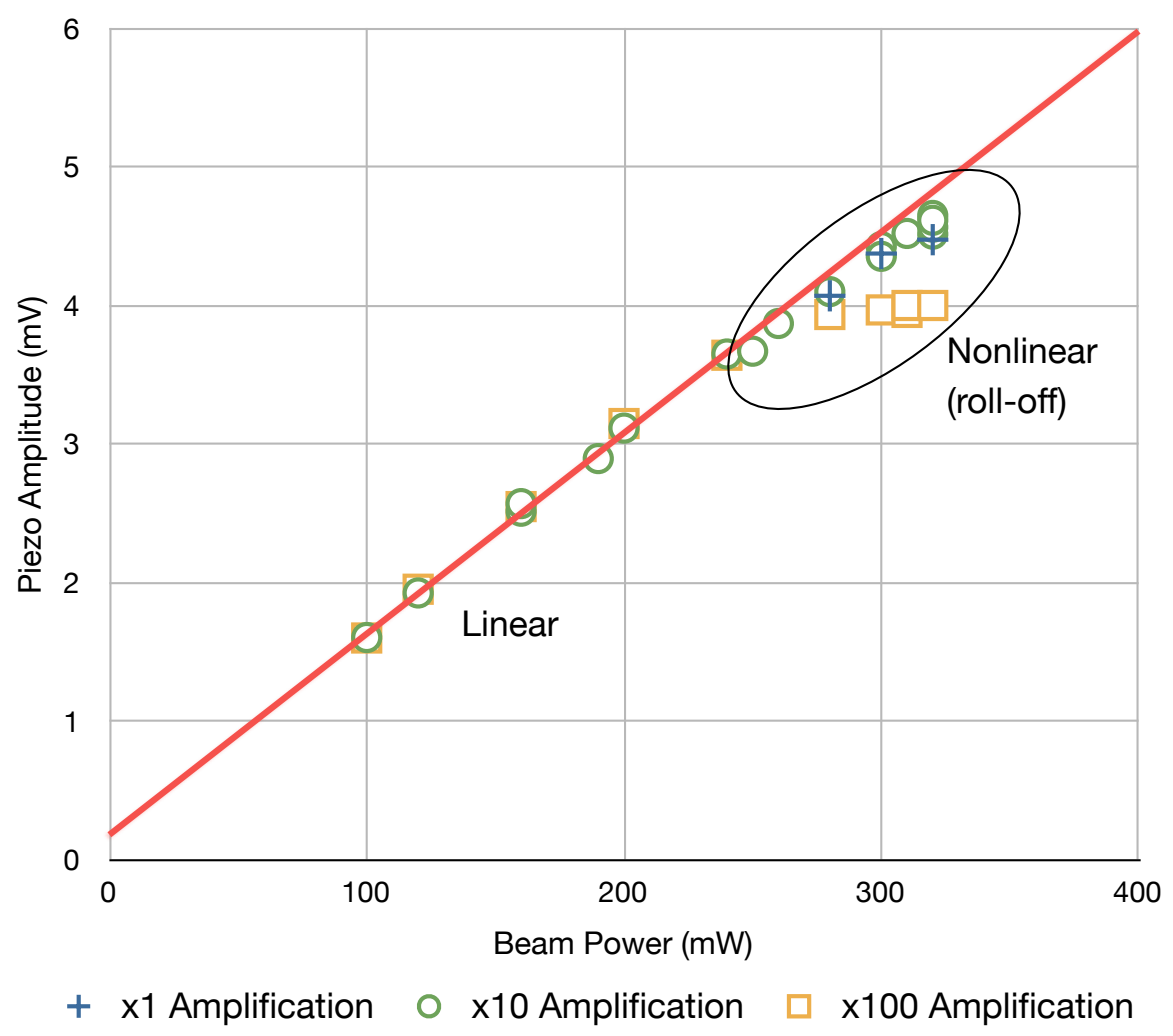

Figure 6: (Color) Plot of vibrational signal amplitude versus beam power for the proof-ofconcept test of the power monitor. The data shows linearity at low beam power (shown by the red trendline), but exhibits nonlinear behavior for pulse energies larger than $2 \mathrm{~mJ}$ (i.e. $240 \mathrm{~mW}$ ). Roll-off is more pronounced for larger amplifications, indicating that amplifier saturation may be a partial cause of nonlinearity. 


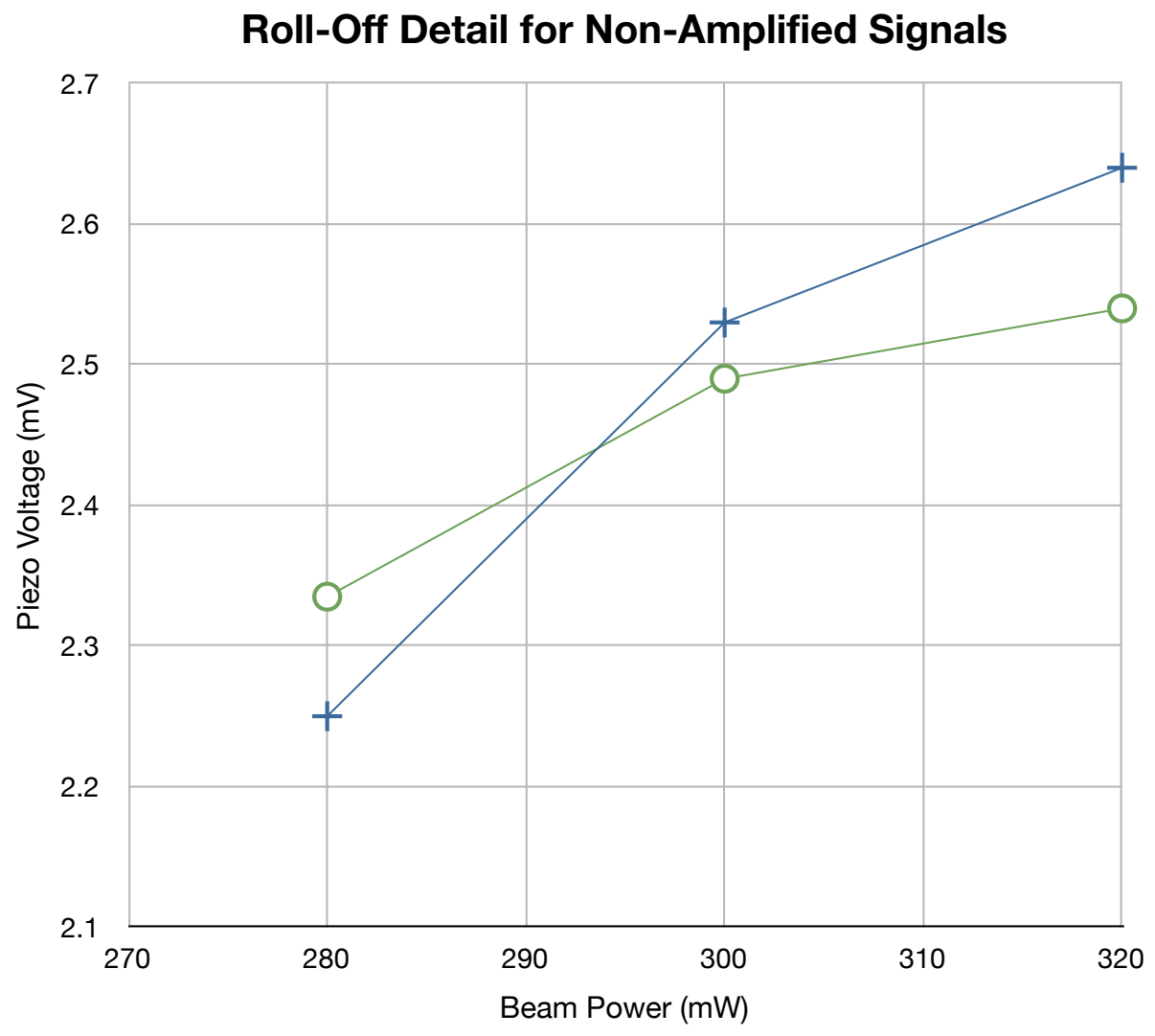

+ Ch. 1 o- Ch. 2

Figure 7: (Color) Plot of vibrational signal amplitude versus beam power for trials in which the amplifier was removed from the signal line. Signal roll-off is still observed, indicating that the phenomena is not due singularly to amplifier saturation. Other effects, still to be studied, must be responsible for the observed roll-off. 


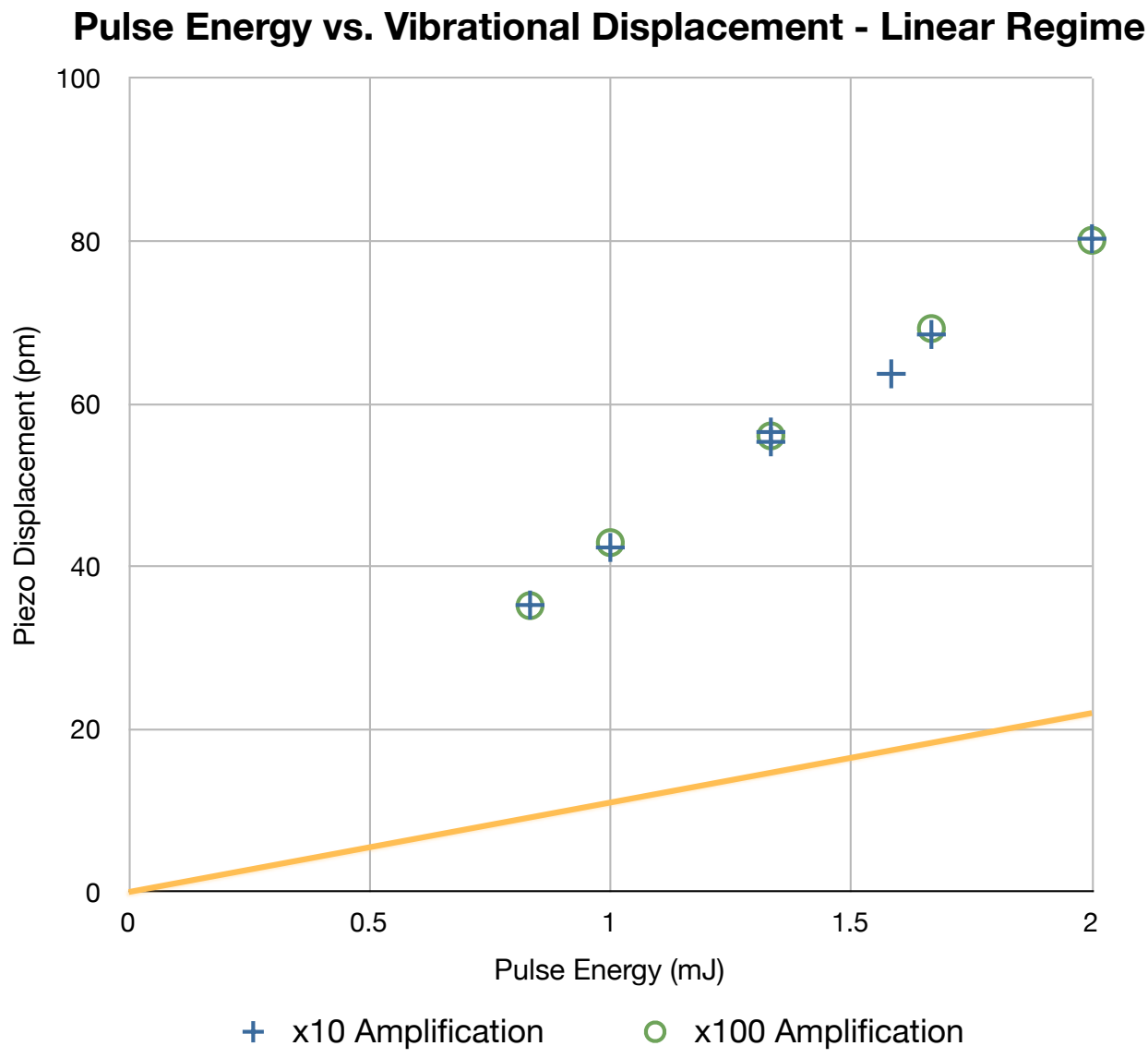

Figure 8: (Color) Plot of vibrational amplitude in the target versus beam pulse energy for the proof-of-concept test. Only data below $2 \mathrm{~mJ}$ are included (i.e. in which linearity is conserved). Theoretical predictions are shown by the yellow line. The slopes of the theoretical and experimental curves differ by a factor of 3 or more, indicating severe errors in the theoretical model. 